\title{
USOS DE LA EVALUACIÓN MEDIANTE PASATIEMPOS COMO ESTRATEGIA DE APRENDIZAJE ACTIVO
}

\section{THE EVALUATION TROUGH HOBBY QUIZZES AS A STRATEGY OF ACTIVE LEARNING}

Sebastián Demyda Peyrás ${ }^{\mathrm{A}}$; María Tania Merinas Amo ${ }^{\mathrm{B}}$; Albano García Sánchez ${ }^{\mathrm{C}}$; Adela González Fernández'; Ana María Melendo Cruz ${ }^{\mathrm{C}}$; Miguel Moreno Millán ${ }^{\mathrm{B}}$ y Ángeles Alonso Moraga ${ }^{*}$

*ge1almoa@uco.es

\section{A Departamento de Producción Animal, Universidad Nacional de La Plata, Argentina}

B Departamento de Genética. Universidad de Córdoba, España

\author{
c Departamento de Historia del Arte, Arqueología y Música. Universidad de Córdoba, España
}

\section{Resumen}

Con el objetivo de mantener el interés en el estudiante y eventualmente autodireccionar el aprendizaje, es preciso modificar, mejorar o ampliar las estrategias o actividades de enseñanza y aprendizaje que cotidianamente se usan en clase. Para ello se propone una herramienta de uso transversal en cualquier disciplina.

Los crucigramas, las sopas de letras y otros entretenimientos intelectuales son un importante recurso en el aula (o fuera de ella), como herramienta educativa y para ampliar la cultura general. Asimismo, constituyen una importante actividad para ocupar el ocio y entretenerse aprendiendo. Los juegos educativos están siendo ampliamente utilizados como diferentes formas de estudio por parte de los estudiantes en diferentes cursos académicos y áreas de estudio y se están convirtiendo en el método más deseado de aprendizaje, ya que hace que la carga de estudio sea menos pesada y que los alumnos disfruten el estudio.

Algunas de las propiedades de la resolución de recursos educativos son:

1. Promueve el dominio específico de un área de conocimiento concreta.

2. Es una forma de estimular el aprendizaje de los estudiantes, que les permite recordar y visualizar la información más relevante de cada materia.

3. Impacta en el desarrollo cognitivo del alumno.

4. Motiva a los alumnos a aprender en lugar de memorizar.

5. Impulsa la confianza del estudiante al obtener respuestas correctas.

6. Es concebido por los estudiantes como un juego o una actividad recreativa.

7. Es una herramienta de aprendizaje efectiva de la terminología, definiciones, ortografía y relación de conceptos clave.

8. Permite a los docentes medir competencias y habilidades alcanzadas por los alumnos.

Por todo ello, la elaboración de un cuaderno de crucigramas y el juego de preguntas Kahoot con distintos niveles de aprendizaje (básico, medio, alto) y estructurado por temáticas, permite a los estudiantes de las distintas asignaturas impartidas por los docentes participantes del presente estudio:

- Motivar el aprendizaje activo de forma entretenida.

- Mejorar el lenguaje escrito (ortografía y vocabulario)

- Mejorar los resultados de la evaluación del conocimiento al familiarizarse con los conceptos clave de cada asignatura y al mejorar la concentración.

Palabras clave: Citogenética; Crucigramas; Evolución; Genotoxicología; Música 


\section{Abstract}

The interest and participation of the students is one of the key points during the development of the learning process. Therefore, all the actions developed in order to increase these two factors during lessons are extremely important in order to achieve a successful learning experience. For this reason, it is necessary to modify, improve or expand teaching strategies and activities that are used during the teacher-student interaction in the classroom every day.

Crossword puzzles, alphabet soups and other intellectually-challenging activities are an important resource in the classroom (or outside of it), both, as an educational tool which can be used to fix specific knowledge and to broaden the general culture of the students. Moreover, they are also described as an important activity which could combine leisure and entertaining with learning. These kind of educational games are being widely used as different forms of study in different academic courses in most of the different knowledge areas and are becoming one of the most desired methods of learning because it makes the study load less cumbersome and highly enjoyable.

Some of the general characteristics of educational resources resolution should be:

1. Promoting the specific domain of an area of concrete knowledge.

2. A way to stimulate student learning, which allows them to remember and visualize the most relevant information in each subject.

3. Impact on the cognitive development of the student.

4. Encouraging students to learn rather than to memorise.

5. Boosting student confidence by getting correct answers.

6. Conceived by students as a play or recreational activity.

7. Effective learning tool for the terminology, definitions, spelling and relation of key concepts.

8. Allowing teachers to measure competencies and skills achieved by students.

Therefore, the aim of this innovation project is the creation of a crossword book and Kahoot-like quiz game with different levels of learning structured across the different course topics which will impact in the student learning process from 3 different scopes:

- Motivate active learning in an entertaining way.

- Improve written language (spelling and vocabulary)

- Improve the results of knowledge assessment by allowing the student to becoming familiar with key concepts of each subject and by increasing their concentration.

Keywords: Cytogenetic; Crosswords; Evolution; Genotoxicology; Music.

\section{INTRODUCCIÓN}

Desde que en el año 1999, en el que España suscribiera junto a otros 29 países la declaración de Bolonia, la Universidad de Córdoba se encuentra ante el reto de adaptar sus sistemas de enseñanza universitaria a este nuevo marco internacional (Fernández-Arévalo et al., 2010). De acuerdo a estas directrices, existentes en el nuevo Espacio Europeo de Educación Superior, todos los alumnos deben adquirir un papel protagonista en el proceso de enseñanza-aprendizaje. Esto se verá reflejado en una serie de cambios destinados principalmente a la consecución de competencias y destrezas, en donde debe potenciarse el aprendizaje autónomo del alumno, preparándolo para que continúe desarrollándose y aprendiendo a lo largo de su vida, proceso conocido como LLL (Long Life Learning).

Los enfoques didácticos más tradicionales utilizados en el aprendizaje son muy amplios y diversos. Entre ellos, podemos nombrar al uso de diferentes paradigmas, teorías, estilos de aprendizaje, actividades basadas en el constructivismo, actividades motivacionales en el aula, así como el desarrollo de las competencias básicas o el uso de las TIC's, etc. (Padial y Gómez, 2013). Estas estrategias de aprendizaje, comprensión y resolución de los mismos pueden ser definidas como las decisiones concretas que adopta el alumno y que permiten una mayor susceptibilidad y permeabilidad hacia el proceso específico de aprendizaje, el cual se vuelve más rápido, fácil, divertido y autónomo. Por este motivo, es cada vez más frecuente en los últimos años, el intentar promover el aprendizaje autónomo entre los alumnos incluyendo métodos de innovación docente, tales como el estudio de casos clínicos, el empleo de recursos periodísticos o el uso de juegos didácticos.

El aprendizaje activo (AA) es aquel que está basado en el alumno, el cual solo puede adquirirse a través de su implicación, motivación, atención y trabajo constante. Las actividades basadas en el AA se caracterizan por ser desafiantes y, por lo tanto, altamente motivadoras, a la vez que permiten profundizar en el conocimiento adquirido, además de desarrollar en los alumnos diferentes habilidades como son la búsqueda, análisis y síntesis de la información. En este sentido, ha sido ampliamente demostrado en estos últimos años que la mayor eficiencia didáctica de los métodos de enseñanza activos frente a los pasivos para desarrollar competencias y lograr aprendizajes significativos y de alto nivel cognitivo (Martí-Parreño et al., 2015). 
Según Freile y Mercado (2000), uno de los campos de estudio en los que la actividad docente se encuentra más retrasada es en lograr una mejora cualitativa y cuantitativa de la transmisión de información desde el docente hacia al alumno. Frente a ello, el uso de diferentes recursos didácticos innovadores tiene una importancia fundamental en la educación, ya que constituyen una importante herramienta facilitadora y altamente motivacional dentro del proceso de enseñanza-aprendizaje. En este sentido, está claro que la metodología tradicional basada en la utilización de clases magistrales y la transmisión verbal y unidireccional del conocimiento posee el inconveniente que, si bien la transmisión puede ser correcta (y no siempre), la recepción e internalización de los contenidos es deficiente, siendo necesaria la inclusión de "favorecedores" de este proceso comunicativo para la mejora cuali-cuantitativa del proceso de aprendizaje. En este sentido, un recurso didáctico muy interesante y cada vez más aplicado son las actividades que involucren juegos de diverso tipo en los cuales el alumnado se vea implicado. Cañete (1993) señala que la presencia de tres aspectos propios relativos al juego, como son el principio del placer, la transformación de lo pasivo en activo y la compulsión y placer de repetición, tienen un efecto altamente motivacional en los seres humanos, lo cual puede resultar fundamental en la mejora del proceso educativo, debido a que sirven como base para fomentar la creatividad e interés de los alumnos.

Los pasatiempos educativos (crucigramas, sopas de letras, autodefinidos, etc.) poseen diversos beneficios cognitivos que los convierten en una herramienta didáctica que desarrolla habilidades específicas y mejora la capacidad de comprensión de las personas que acostumbran resolverlos. Hoy en día, están siendo ampliamente utilizados como formas de estudio auxiliares por parte del alumnado de diferentes áreas de conocimiento, haciendo que el estudio de contenidos sea más placentero para los alumnos, en la mayoría de los casos. (Ogald y Barvadid, 1992) han señalado que la implementación de pasatiempos educativos como parte del proceso de enseñanza-aprendizaje retoma el concepto conocido como "tecnología educativa" el cual se define como "el conjunto de procedimientos o métodos, técnicas, instrumentos y medios, derivados del conocimiento científico, organizados sistemáticamente en un proceso, para el logro de objetivos educativos" desarrollado por Crossman y Crossman, (1983). Más aún, Lomas (1999) afirma que los pasatiempos educativos también influyen en el desarrollo cognitivo y social, además de las habilidades académicas, pues mejoran la atención y concentración, y promueven la búsqueda intensa de estrategias para la solución de problemas, poniendo a trabajar la mente y produciendo un desarrollo de la inteligencia, logrando además mejorar la retención de información y la atención de los alumnos, desarrollar habilidades y destrezas específicas asociadas a la temática de estudio y promover la creatividad y la necesidad de estar informado en ámbitos tanto académicos como culturales. Además, el uso de este tipo de metodologías permite el desarrollo de la capacidad de análisis en períodos breves de tiempo y en condiciones cambiantes, ayuda a fomentar los hábitos y habilidades profesionales para la evaluación de la información técnica y la toma de decisiones colectivas en base al análisis de las mismas (León-González et al., 2010). Pero además, Weisskirch (2006) sostiene que los pasatiempos pueden servir como herramientas para que los estudiantes puedan medir el grado de comprensión sobre algún tópico particular, a modo de guía de autoevaluación, que les permitirá tener una noción "a la carta” de la comprensión e incorporación de dichos contenidos, lo cual promueve aún más la participación activa de los estudiantes en el proceso de enseñanzaaprendizaje. El beneficio del uso de pasatiempos educativos como herramientas didácticas en el proceso de enseñanzaaprendizaje ha sido evidenciado por numerosos autores (Childers, 1996; Silberman, 1996; McKeachie, 2002; Franklin et al., 2003; Lewis, 2003; Castañeda, 2005; Weisskirch, 2006; Olivares et al., 2008; Akl et al., 2010).

En el presente estudio, uno de las asignaturas analizadas fue la genética veterinaria, la cual es un área dentro de la licenciatura en Veterinaria que suele chocar con la falta de interés del alumnado. En general, el perfil del veterinario, tanto de grandes como de pequeños animales, tiende a estar más enfocado hacia la clínica, la cirugía, el manejo de enfermedades o la seguridad alimentaria, lo que hace que el alumnado perciba la asignatura, probablemente de forma errónea, como accesoria y sin una gran importancia para el desarrollo de su carrera. Más aún cuando parte de la misma se basa en el análisis de modelos matemáticos complejos, de los cuales los estudiantes de ciencias naturales suelen rehuir. Algo similar suele suceder en el ámbito de la música, más aún cuando ésta forma parte de carreras universitarias no específicas, en las cuales también es considerada como una asignatura secundaria. La implementación de pasatiempos educativos en este tipo de áreas del conocimiento, puede resultar en una forma válida de potenciar el aprendizaje activo de forma entretenida y mejorando los resultados educativos. Mediante su uso el alumnado podrá familiarizarse con los conceptos clave de cada una de ellas, que además poseen una elevada carga teórica y una constante evolución y complejidad, tornándose dificultosas en algunos casos. Por tanto, el profesorado de este tipo de asignaturas debe procurar introducir nuevas metodologías docentes, entre las que se encuentran las analizadas en el presente estudio, que les permitan a los alumnos llevar adelante un aprendizaje más activo facilitando su aprendizaje autónomo de forma divertida.

Entre los beneficios específicos que se intentan lograr, particularmente en el proceso de educación superior, mediante el uso de estas metodologías, Cedeño, (1995) y Olivares et al., (2008) han postulado los siguientes:

- Aumentar el interés de los estudiantes y su motivación por las asignaturas técnicas.

- Comprobar el nivel de conocimiento técnico alcanzado por los estudiantes.

- Tener un alto grado de interés para los estudiantes.

- Estimular la actividad por parte de los alumnos.

- Desarrollar habilidades generalizadas y capacidades profesionales.

- Contribuir al aumento de los significados y al desarrollo del vocabulario. 
- Proporcionar una base concreta para el pensamiento conceptual.

- Mejorar las relaciones interpersonales y hacer más amenas las clases.

- Aumentar el nivel de preparación.

- Hacer que el aprendizaje sea más duradero.

- Desarrollar continuidad de pensamiento.

- Contribuir a la eficiencia, profundidad y variedad del aprendizaje.

\section{Objetivos propuestos}

El objetivo principal perseguido en este estudio ha sido evaluar el uso de pasatiempos con fines educativos dentro de los curricula abarcados (Evolution del Grado en Biología, Genética del Grado en Veterinaria, Genotoxicología del IDEP y Desarrollo de la Expresión Musical en Infantil). Para ello se han diseñado pasatiempos específicos (crucigramas, sopas de letras), así como plantillas de evaluación en tiempo real mediante el uso del teléfono móvil como herramientas didácticas para amenizar la enseñanza, incrementar el interés por el aprendizaje de los alumnos y fortalecer la incorporación de conceptos fundamentales de cada una de las asignaturas y temáticas abarcadas. Adicionalmente, y con el fin de fomentar el plurilingüismo, el presente estudio se ha realizado en asignaturas impartidas en ambos idiomas.

De manera secundaria, nuestro estudio ha buscado evaluar si el uso de estas metodologías transformaba al estudiante en agente activo durante las clases, a diferencia de lo buscado con el método memorístico normalmente utilizado en el proceso tradicional, en el cual el alumno es considerado un sujeto pasivo.

Por último, nuestro estudio propuso analizar en tiempo real la participación del alumno y el grado de entendimiento de los conceptos vertidos en la clase a través de la realización de exámenes interactivos mediante el uso de sus respectivos teléfonos móviles.

Entre las competencias a desarrollar por el alumnado que se hemos evaluado se encuentran:

- La comunicación escrita

- La capacidad de sintetizar conceptos

- La competitividad y motivación por el éxito

En definitiva, los profesores implicados en la impartición de la docencia en cada una de las asignaturas citadas han podido analizar el efecto de la influencia que ha tendido la utilización de estos recursos educativos sobre el aprendizaje logrado.

\section{Material y Métodos}

Para la confección de los pasatiempos, y después evaluar diversos programas existentes de uso libre en la red, se seleccionaron dos softwares de fácil manejo que permitieron generar el material didáctico del estudio (sopas de letras, crucigramas y encuestas virtuales interactivas mediadas por el teléfono móvil u ordenadores personales) y que además presentaron una amplia variabilidad de funciones. Estos han sido:

- Crossword compiler (http://www.crossword-compiler.com/) y

- Kahoot (https://www.kahoot.com).

\subsection{Utilización de sopas de letras (CROSSWORD COMPILER)}

El primer paso ha sido confeccionar, por asignatura y por área, una base de datos de preguntas-respuestas para cada uno de los tópicos a desarrollar, lo cual fue realizado de acuerdo con los docentes a cargo de cada una de las asignaturas participantes del estudio. A partir de la misma se diseñaron pasatiempos personalizados, seleccionando las preguntas más adecuadas en función del nivel de conocimientos, el objetivo de evaluación, las características del alumnado, los contenidos de la enseñanza y el ritmo de aprendizaje (Imagen 1). Todas estas actividades fueron confeccionadas tanto en inglés como en castellano, de acuerdo con la pertenencia o no de cada asignatura al plan de plurilingüismo. Adicionalmente, parte de los contenidos estuvieron disponibles en ambos idiomas, permitiendo al alumno el poder comparar nomenclaturas y conceptos entre ellos. 


\section{FENÓMENO SONORO Y ANATOMÍA DEL OÍDO}

Encuentra 30 palabras relacionadas con el fenómeno sonoro y la anatomía del oído.

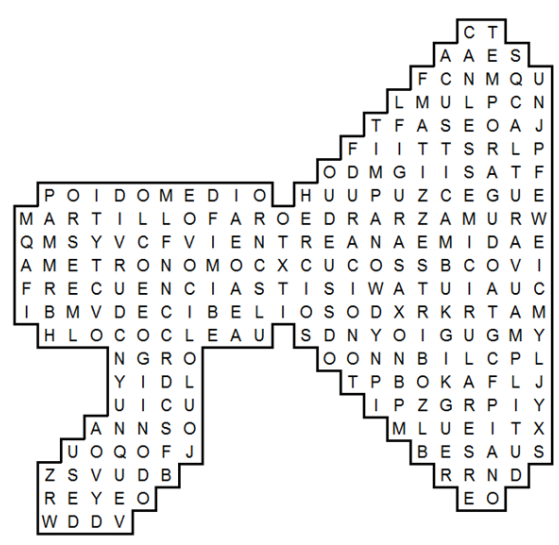

\section{EVOLUTION}

Find 30 words related with Evolution.

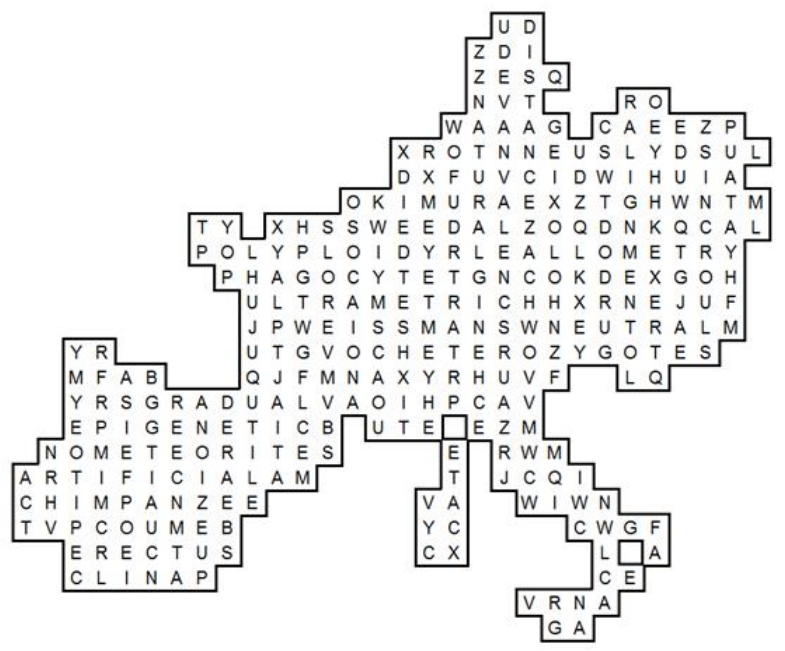

Imagen 1. Ejemplos de pasatiempos.
ENSAYOS GENOTOXICOLÓGICOS EN SISTEMAS MODELO

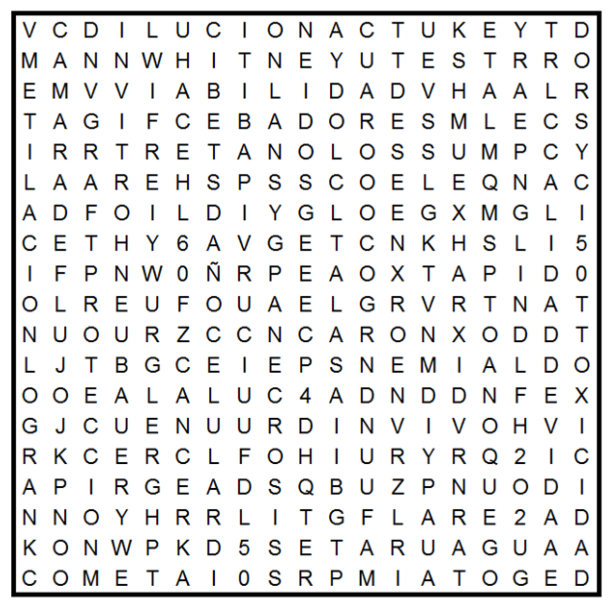

CYTOGENETIC
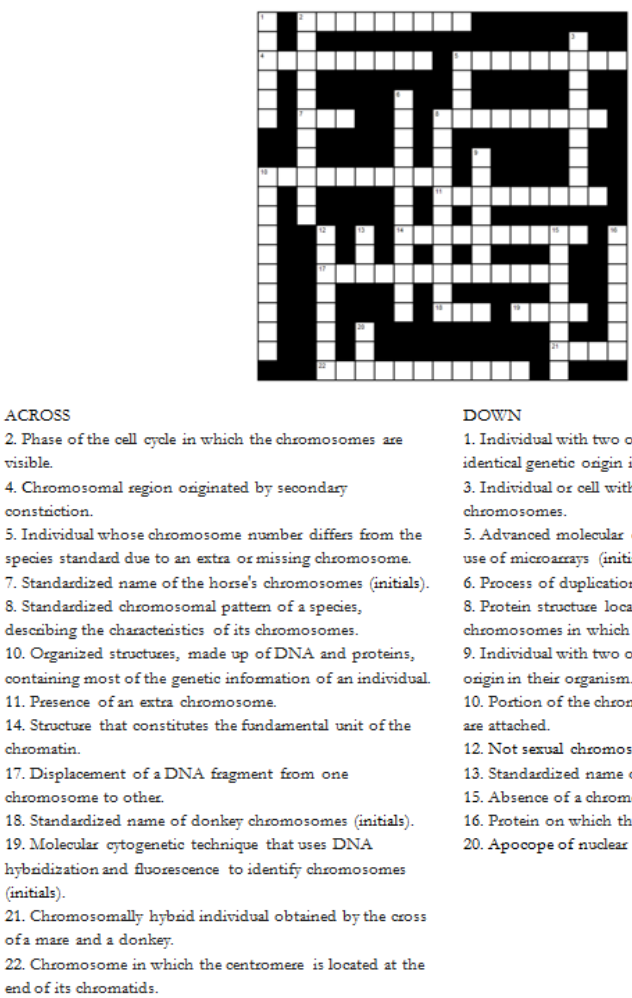

Doris

1. Indiridual with two or more different cell lines of identical genetic origin in their organism. 3. Indiridual or cell with three or more complete sets of chromosomes.

Advanced molecular cytogenetic technique based on the use of microarrays (initials).

6. Process of duplication and division of genetic material. chromosomes in which the microtubules are anchored. 9. Individual with two or more cell lines of different genetic origin in their organism.

10. Portion of the chromosome in which both chromatids are attached. 13. Standardized name of borine chromosomes (initials). 15. Absence of a chromosome in one of the pairs. 16. Protein on which the DNA strand is rolled up. 20. Apocope of nuclear organizer. 8. Protein structure located near to center of the

12. Not sexual chromosome

Los diferentes pasatiempos educativos se estructuraron en distintos niveles de complejidad, pudiendo el estudiante elegir el pasatiempo a resolver según el nivel de aprendizaje que creía poseer. Esto permitió también realizar una autoevaluación de los conocimientos adquiridos. Todos estos pasatiempos fueron incluidos en un documento específico para cada asignatura, los cuales fueron testados de manera preliminar entre grupos de alumnos de las mismas. Adicionalmente, se confeccionó una guía de soluciones de los ejercicios propuestos en formato digital (Imagen 2), la cual posibilitó que el alumno pudiera comprobar si la resolución de la actividad de su pasatiempo fue correcta, así como dar respuesta a aquellas definiciones que no hubiera sabido solventar. Estas soluciones fueron entregadas al alumnado una vez que el profesor evaluó el grado de éxito alcanzado por los alumnos en la resolución de los pasatiempos. 


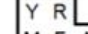

FENÓMENO SONORO Y ANATOMÍA DEL OÍDO

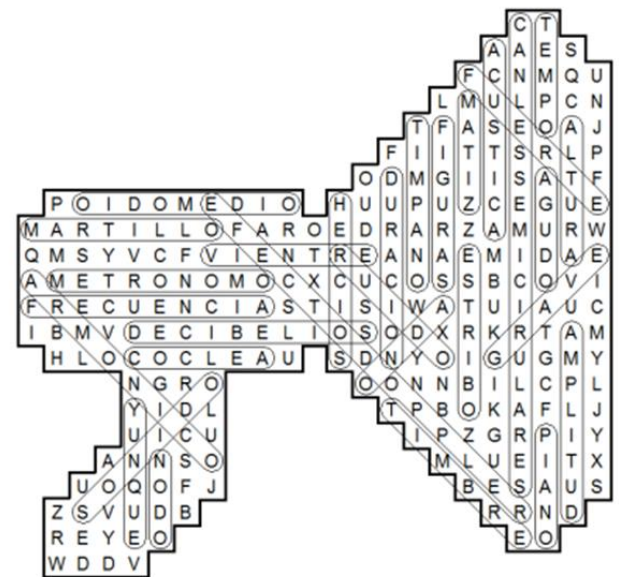

EVOLUTION

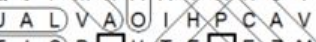

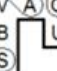

EDE Z $T$ M
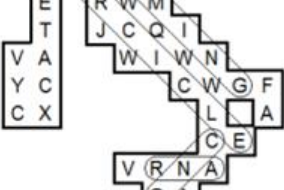

P

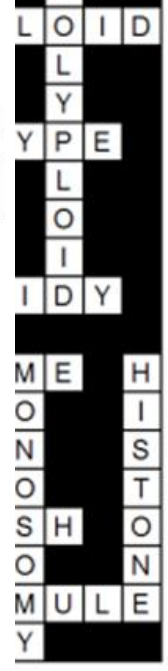

ENSAYOS GENOTOXICOLÓGICOS EN SISTEMAS MODELO

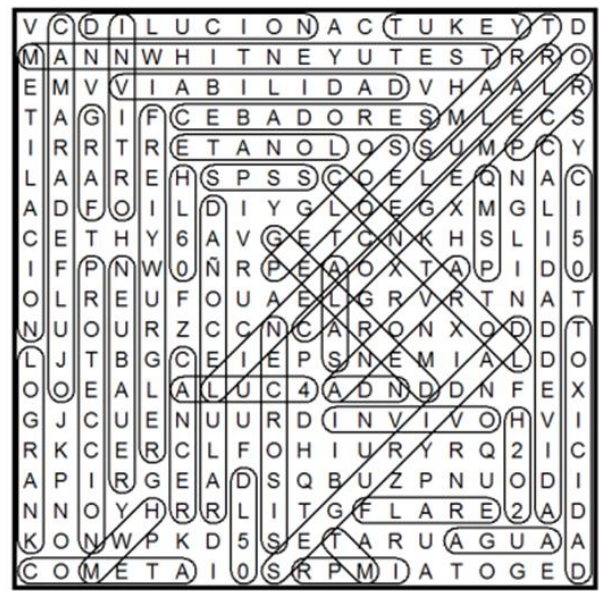

Imagen 2. Soluciones de pasatiempos.

\subsection{Evaluaciones en tiempo real mediante el uso de teléfonos móviles (KAHOOT)}

La utilización de cuestionarios virtuales en tiempo real mediante el uso del teléfono móvil durante la clase es una forma extraordinaria para "gamificar" el aprendizaje y hacer que los alumnos aprendan divirtiéndose. Existen diversas aplicaciones que permiten este tipo de evaluaciones en tiempo real, siendo la aplicación KAHOOT, la más simple e interactiva, desde nuestro punto de vista. La misma permite crear cuestionarios de preguntas con respuestas múltiples predeterminadas, las cuales son formuladas de manera secuencial y en un orden establecido, y son respondidas por el alumnado, mediante una aplicación instalada en su teléfono móvil, en tiempo real a modo de competencia o juego. Inicialmente, cada uno de los profesores implicados en la docencia incluida en el estudio creó una base de datos de preguntas-respuestas a través de la página web de la aplicación (www.getKahoot!.com), mediante la cual se pueden desarrollar test específicos, determinar su duración o el tiempo de usadopara responder cada pregunta, la posibilidad de formar equipos, el tiempo para cada respuesta, etc. En dicha base de datos se incluyeron diferentes tipos de preguntas, adecuadas en función de la asignatura, el nivel de conocimientos, el objetivo de evaluación, las características del alumno, los contenidos de la enseñanza y el ritmo de aprendizaje, tanto en idioma castellano como en idioma inglés. Dichos cuestionarios fueron además completados mediante la utilización de recursos multimedia (imágenes, gráficas, vídeos, etc.) que ponen en contexto la pregunta y aumentando la motivación y novedad a la hora de realizar el juego, tanto para el profesorado como para el alumnado (Imagen 3). 


\section{¿Qué modelo animal se usa en los ensayos in vivo?}

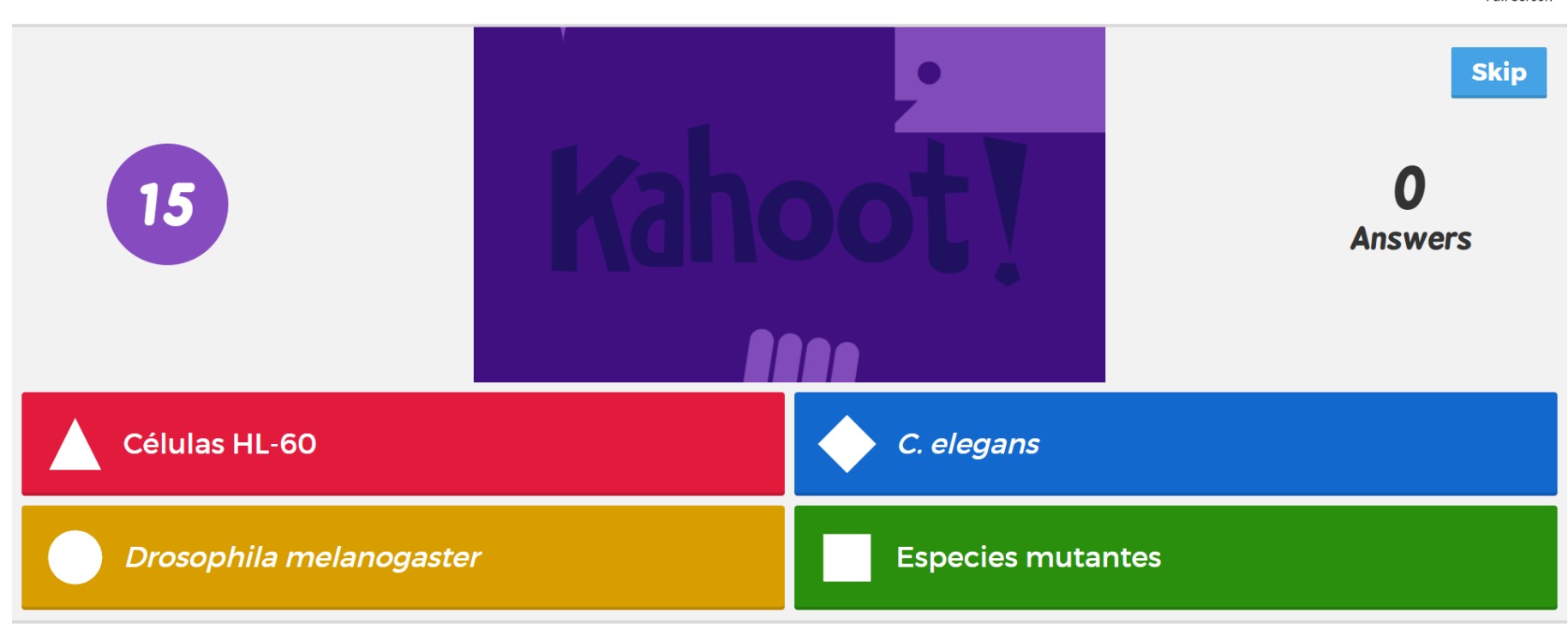

\section{kahoot.it Came PIN: 1415955}

Imagen 3. Ejemplo de preguntas creadas en Kahoot

La capacidad motivacional de este recurso fue probada de manera preliminar con grupos de estudiantes de las clases de las diversas asignaturas incluidas en el estudio. Cada profesor dispuso de un ordenador conectado a la pantalla en la que los alumnos, una vez comenzada la actividad, observaron la aparición de las preguntas de manera ordenada y sus posibles respuestas, teniendo un tiempo limitado preestablecido para responderlas. Las preguntas fueron contestadas mediante los dispositivos móviles de cada alumno, los cuales debieron haberse previamente registrado mediante la aplicación propia y un código proporcionado por el docente para cada test específico. Además, las posibles respuestas fueron también identificadas según un código de colores; debiendo los alumnos elegir la correcta entre todas ellas (Imagen 4).
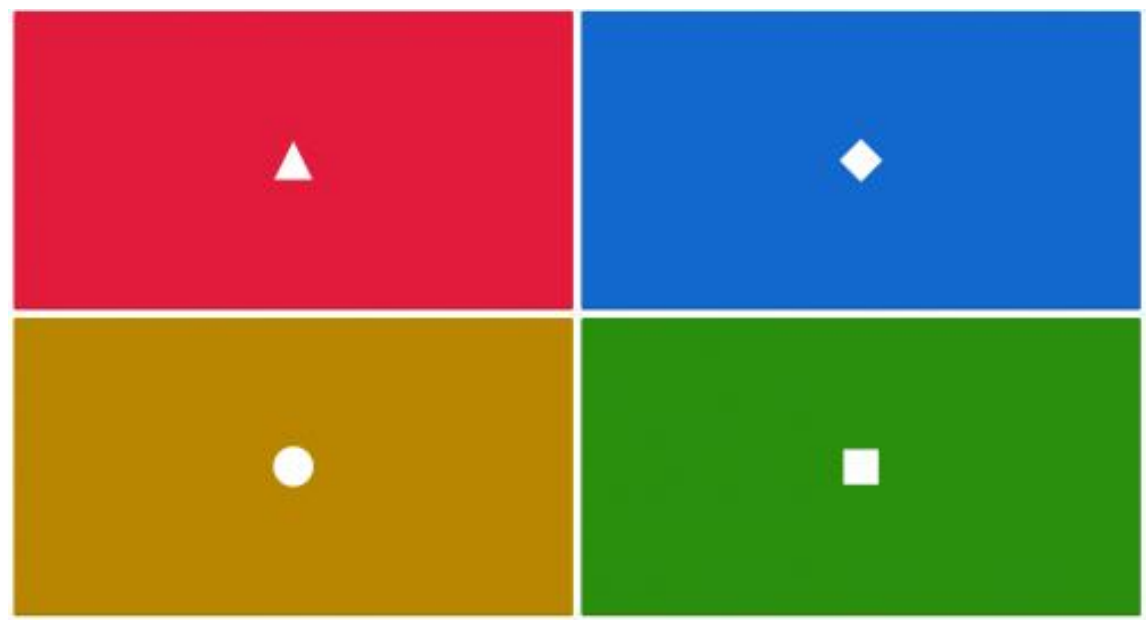

Imagen 4. Código de respuestas para las preguntas creadas en Kahoot.

Al terminar la partida se confecciona, de manera automática por el programa, un listado con las puntaciones obtenidas durante el juego por los diferentes participantes, consiguiendo puntos ficticios por haber respondido correctamente, pero también debido al tiempo en el que lo han hecho (a menor tiempo mayor puntaje). Además, el programa realizó un ranking de los estudiantes, así como un podio de triunfadores en el cual se identifica a los mejores participantes. Adicionalmente, el mismo programa generó un reporte en formato Excell ${ }^{\mathrm{TM}}$ descargable mediante el cual queda registro de la participación de cada estudiante, su desempeño y de la dificultad o compresión de cada una de las preguntas. Gracias a ello el profesorado dispone de todo tipo de datos y estadísticas que pueden servirle para conocer la evolución de los estudiantes. 


\section{Resultados obtenidos y Discusión}

Los pasatiempos educativos están demostrando ser una herramienta valiosa para el profesorado que permite una mayor interacción con el alumno en sus funciones específicas de planificación, conducción y evaluación de las clases y asignaturas. Son además de una ayuda orientada a facilitar la intervención pedagógica del profesorado en las aulas y el aprendizaje del alumnado, así como una expresión de una determinada concepción de la enseñanza y del aprendizaje en sí mismos (Lomas, 1999). Ha sido demostrado que su uso influye en el desarrollo cognitivo y social, así como en las habilidades académicas, mejorando la atención y concentración, y promoviendo la búsqueda intensa de estrategias para la solución de problemas, lo cual produce un aumento de la actividad cognitiva y, finalmente, un mayor desarrollo de la inteligencia en el proceso de enseñanza-aprendizaje (León-González et al., 2010).

Los resultados obtenidos de manera preliminar utilizando, pasatiempos y encuestas interactivas en tiempo real (Kahoot) con grupos de alumnos pertenecientes a las distintas asignaturas de las áreas de Genética y Música han sido prometedores. Se ha conseguido un alto grado de participación en las experiencias piloto realizadas, mostrando el alumnado un renovado interés por temas ya dictados. La resolución de los ejercicios gráficos se ha llevado a cabo en tiempo y forma por parte del alumnado participante. Por otro lado, los cuestionarios interactivos han sido más prometedores aún, con una alto grado de interés, de atención y predisposición para la realización de los mismos, permitiendo al profesorado detectar fallos en la comprensión de ciertos contenidos que pudieron volver a ser explicados o corregidos durante las clases de manera satisfactoria. Cabe destacar que en el presente trabajo, el reducido número de alumnos involucrados ha impedido el realizar valoraciones cuantitativas respecto a os resultados, entendiéndose el mismo como una experiencia piloto o preliminar. Sin embargo los resultados cualitativos recogidos por los autores han demostrado u alto grado de cumplimiento y de interés de los alumnos, con un elevado grado de participación. Un resumen de los aspectos favorables y desfavorables encontrados durante el desarrollo de los juegos educativos se detalla en la Tabla 1.

\begin{tabular}{|c|c|c|}
\hline & Favorable & Desfavorable \\
\hline $\begin{array}{l}\text { Crossword } \\
\text { compiler }\end{array}$ & $\begin{array}{l}\text { - Recurso tangible y portable } \\
\text { - Concreción de las cuestiones } \\
\text { - Ampliación del vocabulario } \\
\text { - Fijación de terminología específica }\end{array}$ & $\begin{array}{l}\text {-Recurso individual } \\
\text {-Demasiado constreñido a respuesta de una } \\
\text { solución } \\
\text { - Falta de interés de cierta parte del alumnado }\end{array}$ \\
\hline Kahoot & $\begin{array}{l}\text {-Resultados obtenidos en directo } \\
\text {-Posibilidad de discutir/comentar las respuestas } \\
\text {-Recurso dinámico grupal }\end{array}$ & $\begin{array}{l}\text {-Necesidad de recursos informáticos } \\
\text {-Solo se puede realizar en clase }\end{array}$ \\
\hline
\end{tabular}

\section{Tabla 1: Análisis cualitativo de los resultados obtenidos}

Como indican Martí-Parreño et al., (2015), "una de las consecuencias más dolorosas y perniciosas del desinterés y falta de motivación de los estudiantes con su educación es el abandono de los estudios. Parte de esta falta de interés y motivación en los estudiantes actuales puede estar determinada por el rol pasivo que desempeña el estudiante en las metodologías de enseñanza tradicionales". Por ello, consideramos que el uso de recursos educativos como complemento para la clase donde el alumno participe de forma dinámica y divertida, junto con la labor docente habitual, favorece el aprendizaje y motivación del estudiante, especialmente de aquellos alumnos considerados nativos digitales (Prensky, 2001), que son los más dados a reclamar esta interacción en el aula.

Este estudio ha estado basado en el principio de gamificación, definida por Deterding et al., (2001) como "el uso de los elementos del diseño de juegos en contextos que no son juegos". Además, el uso de juegos interactivos mediados por el móvil permite al profesor evaluar de forma continuada si los contenidos o competencias trabajadas están siendo adquiridos de manera correcta y completa. Adicionalmente, la simple observación de las pruebas realizadas y la naturalidad de presentar las cuestiones conducirán al alumno a una evaluación exenta de presión y ansiedad, permitiendo una mejor resolución de los conflictos y problemas creados por la evaluación que podrían ser resueltos con mayor facilidad en el seno del juego, de manera amena, rápida y colaborativa. Es decir, los pasatiempos educativos han resultado ser una herramienta excelente en el proceso de aprendizaje del alumno y al mismo tiempo los profesores pueden utilizarla como una forma de evaluación del aprendizaje nada tradicional.

\section{Conclusiones}

A lo largo del proceso se ha pretendido no solo involucrar al alumnado en el proceso de enseñanza-aprendizaje para lograr una mayor participación y mejores resultados, sino también introducir el uso de pasatiempos como herramienta metodológica de una forma interdisciplinar y transversal. Esto ha quedado demostrado en el hecho que las mismas herramientas pueden ser utilizadas en ramas tan diferentes de la enseñanza como la música o la genética veterinaria. Pero 
además, el hecho de que las herramientas puedan utilizarse en diferentes idiomas refuerza su potencial pedagógico en la utilización del inglés como primera lengua extranjera.

Los resultados obtenidos con la utilización de las herramientas de diseño de crucigramas y sopas de letras (Crossword Compiler), así como de Kahoot, demuestran que, efectivamente, el interés y la participación del alumnado han aumentado en las distintas asignaturas, lo que nos lleva a pensar que, como futuras líneas de acción, sería interesante ampliar este tipo de actividades a otras materias y titulaciones que también pudieran beneficiarse de ellas. Consideramos también un aspecto de mejora la utilización de una mayor diversidad de herramientas, así como su posible inclusión en las actividades de evaluación de las asignaturas y en la metodología de trabajo colaborativo.

\section{Agradecimientos}

Agradecemos a los estudiantes que han participado en esta experiencia piloto, sin los cuales no podría haberse llevado a cabo. Agradecemos también a la Universidad de Córdoba por haber financiado parcialmente el desarrollo de este estudio mediante su Plan de Mejora en la Calidad Docente.

\section{Bibliografía}

Akl, E., Pretorius, R., Sackett, K., Erdley, W., Bhoopathi, P., Alfarah, Z., Schünemann, H. "The effect of educational games on medical student's learning outcomes: a systematic review". Medical Teacher, 2010/32 (1), P .16.

Cañete, H. "Juego y vida". El Ateneo, Buenos Aires, Argentina:,1993.

Castañeda, J. “Tus hobbies también sirven”. Periódico "El Norte", México, 2005/14 de noviembre.

Cedeño, M. "Juegos y materiales didácticos para la educación integral en salud de niños y adolescentes". Pedagogía, 1995/95.

Childers, C.D. "Using crossword puzzles as an aid to studying sociological concepts". Teaching Sociology, 1996/24, P. 231-235.

Crossman, E.K., Crossman, S.M. "The crossword puzzle as a teaching tool". Teaching of Psychology, 1983/10, P. 98-99.

Deterding, S., Dixon, D,. Khaled, R., Nacke, L. "Gamification: toward a definition". In Chi 2011 Gamification workshop proceedings, 2011, Vancouver, Bc, Canada.

Fernández-Arévalo, M., Delgado, M.O., García-López, I., García-Márquez, J.A., Saborido, A., Vega-Pérez, J.M. "Impacto del plan piloto para el EEES en la facultad de farmacia de la universidad de Sevilla". Ars Pharm, 2010/51 (2), P. 479-486.

Franklin, S., Peat, M., Lewis, A. "Non-traditional interventions to stimulate discussion: the use of games and puzzles". Journal of Biologycal Education, 2003/37, P. 79-84.

Freile, I., Mercado, J. "Representación gráfica del movimiento: desarrollo interdisciplinar en las áreas de educación física y plástica". Tándem. Didáctica de la Educación Física, 2000/1, P.101-106.

León-González, A.J., Martín-Cordero, C., Sánchez-Hidalgo, M. "El uso de crucigramas como método de innovación docente en la asignatura de farmacia clínica". Actualidad en Farmacologia y Terapéntica, 2010/10 (1), P. 69-71.

Lewis, P. "Is this going to be on the test? in essays from excellence in teaching" In W. Buskist, V. Herven And G.W. Hill (Ed.), USA, 2003/Iv.

Lomas, C. "Cómo enseñar a hacer las cosas con las palabras: teoría y práctica de la evaluación lingüística" In Piedós Ibérica (Ed.), Volumen I, Barcelona, 1999.

Martí-Parreño, J., Quero-Ameijeiras, C., Méndez-Ibáñez, E., Giménez-Fita, E. "El uso de la gamificación en la educación superior". In XII Jornadas internacionales de innovación universitaria. educar para transformar: aprendizaje experiencial, 2015, P. 95-102.

Mckeachie, W.J. "Teaching tips: strategies, research, and theory for college and university teachers" In W.J. Mckeachie (Ed.), Boston, 2002.

Ogald, I., Barvadid, E. "Los materiales didácticos: medios y recursos de apoyo a la docencia" In Trillas (Ed.), México, 1992. 
Olivares, J.C., Escalante, M., Escarela, R., Campero, E., Hernández, J.L, López, I. "Los crucigramas en el aprendizaje del electromagnetismo". Rev. Eureka Enseñ. Divul. Cien., 2008/5 (3), P. 334-346.

Padial, S., Gómez, M.J. "Aprender jugando: una forma divertida y práctica de aprender" In Uned (Ed.), Libro de Actas de Las I Jornadas internacionales de innovación docente dniversitaria en entornos de aprendizaje enriquecidos, Madrid, 2013, P. 156-157.

Prensky. M. (2001). Digital game-based learning. New York: Mcgraw-Hill.

Silberman, M. "Active learning: 101 strategies to teach any subject" In W.J. Mckeachie (Ed.), Boston, 1996.

Weisskirch, R. "An analysis of instructor-created crossword puzzles for student review". Collage Teaching, 2006/54 (1), P. $198-202$. 